\title{
Homogeneous Nucleation of Water Anomalies Explained by Recognition and Elimination of Heterogeneous Species
}

\author{
Donald E. Hagen, James L. Kassner, JR., and Ronald C. Miller ${ }^{1}$ \\ Physics Department and Graduate Center for Cloud Physics Research, University of Missouri-Rolla, Rolla 6540I
}

(Manuscript received 27 October 1981, in final form 11 January 1982)

\begin{abstract}
A piston-type expansion cloud chamber, capable of producing short pulses of nucleation, was used to study the homogeneous nucleation of water over a wide range of temperature and supersaturation. A large effort was made to remove impurities capable of acting as heterogeneous nuclei from the system. The chamber was found to be capable of performing experiments that were relatively free of interfering impurities and the results resolved several anomalous nucleation phenomena appearing in the literature: the knee in drop concentration vs supersaturation data, the temperature dependence of the critical supersaturation, and the decay of the nucleation rate with time. The anomalies were found to be due to impurities active at different temperature and supersaturation regimes.
\end{abstract}

\section{Introduction}

A comprehension of the nucleation process is a key element in the understanding of many natural and industrial processes. It figures prominently in gas-toparticle conversion and in unraveling the complex manner in which silver iodide and other artificial nucleants induce ice nucleation in supercooled cloud. Nucleation from the vapor phase is perhaps the easiest phase transition to study and homogeneous nucleation is the simplest form of nucleation to investigate. All theories of nucleation borrow heavily on concepts developed for homogeneous vapor phase nucleation, giving it a position of central importance. In spite of considerable effort, studies of nucleating systems have been fraught with difficulty so that, overall, progress has been slow. Advances have generally resulted from improved technology and better understanding of the methods of controlling competing heterogeneous nucleation processes resulting from minority impurities or unwanted reactive species in the system.

Since the innovative work of Wilson (1897), the expansion cloud chamber has been developed and used by numerous investigators to study homogeneous nucleation (Hirth and Pound, 1963), in many cases taking advantage of technology advances made in nuclear physics applications of the expansion chamber (Das Gupta and Gnosh, 1946). In particular, Kassner and his co-workers (Allard and Kassner, 1965; Kassner and Schmitt, 1966; Kassner et al., 1968; Allen and Kassner, 1969; Anderson et

\footnotetext{
${ }^{1}$ Present affiliation: Central State University, Edmond, OK.
}

al., 1980) developed numerous advances in expansion chamber technology and applied them to a variety of nucleation and condensation phenomena.

In this paper we report on the use of one of our expansion cloud chambers to resolve three anomalous nucleation phenomena appearing in the literature: 1) the "knee" in the nucleation data (drop concentration vs supersaturation) reported by Allen and Kassner (1969) and Biermann (1971); 2) the temperature dependence of the critical supersaturation required for the onset of nucleation at low temperatures (Madonna et al., 1961); and 3) the decay of the nucleation rate for water with time observed by Allard and Kassner (1969) and Biermann (1971). In all cases the anomalies are believed to be due to impurities causing heterogeneous nucleation effects rather than to new facets of the homogeneous nucleation phenomenon.

\section{Experimental apparatus and procedure}

The basic components of the expansion cloud chamber are shown in Fig. 1, and a functional block diagram is given in Fig. 2. This type of automated expansion cloud chamber is described in detail by Kassner et al. (1968) and Schmitt (1981). All of the supporting subsystems (data acquisition and control, optics, pressure measurement, temperature measurement and control, and environmental housing) are either identical or very similar to those described by Schmitt (1981) for his chamber used for nucleation studies on organic compounds, and the reader is referred there for details.

The present cloud chamber has incorporated a 


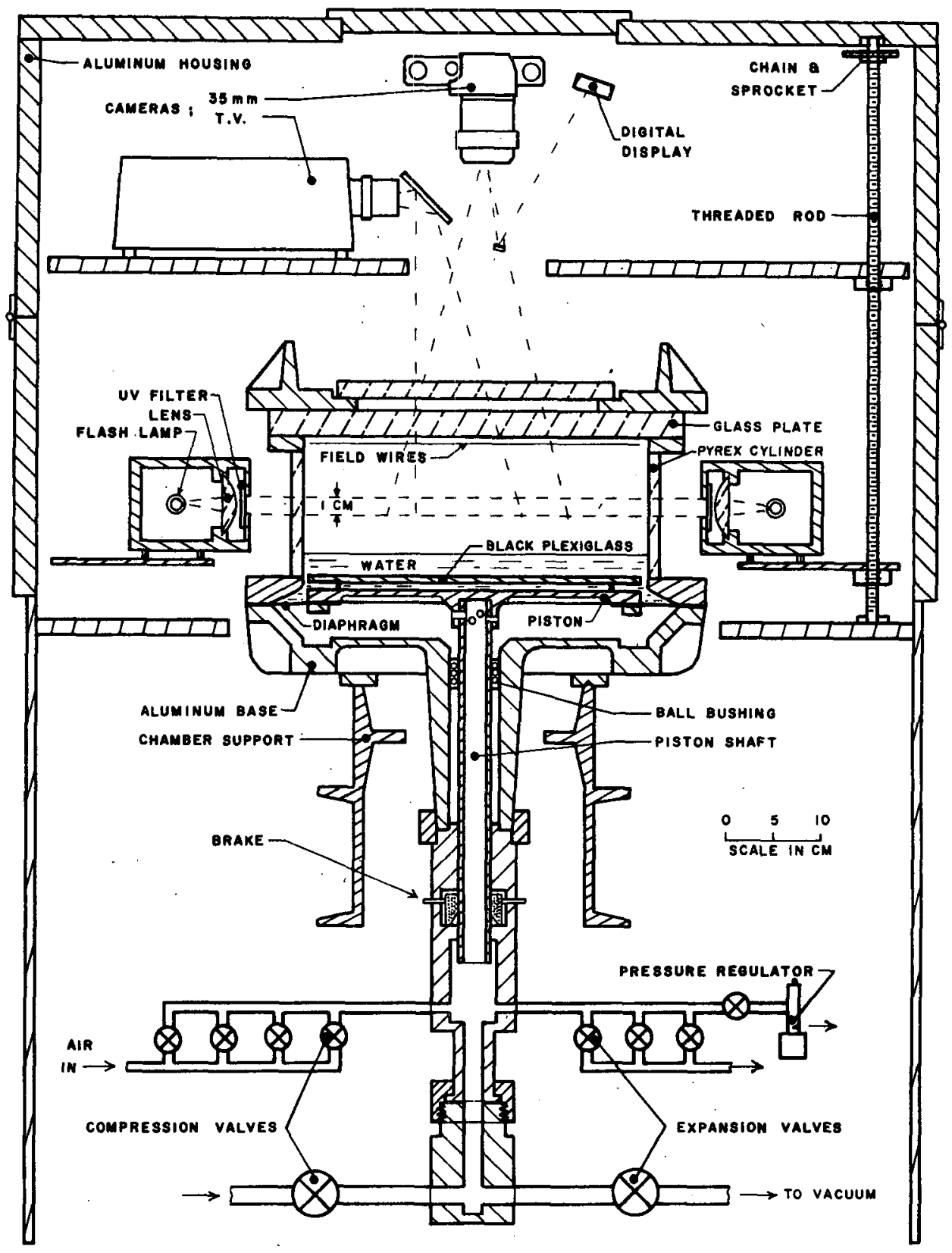

FIG. 1. The expansion chamber used for homogeneous nucleation of water studies.

number of useful features. The diameter of the chamber has been increased over the one used by Allard and Kassner (1965), Allen and Kassner (1969), and Biermann (1971) to give a larger volume-to-surface ratio which provides somewhat longer times before wall effects propagate into the sensitive experimental volume. Major improvements were made in the temperature controller, so that conditions before the expansion are more easily controllable and can be changed with much less effort. The entire apparatus is located in a well insulated walk-in environmental chamber capable of temperatures well above and below room temperature. Needless to say control and data acquisition equipment is located outside the environmental chamber. The temperature control on the cloud chamber itself is by means of heaters which raise the apparatus temperature slightly above that of the environmental chamber. This allows investigations over a wide range of temperatures. The chamber facility proved to be so reliable that it could be left running continuously with minimial supervision. A vertical electric field of $\sim 70 \mathrm{~V} \mathrm{sm}-1$ is set up between the field wires and the wates: pool and is used to sweep ions from the chamber, which is 


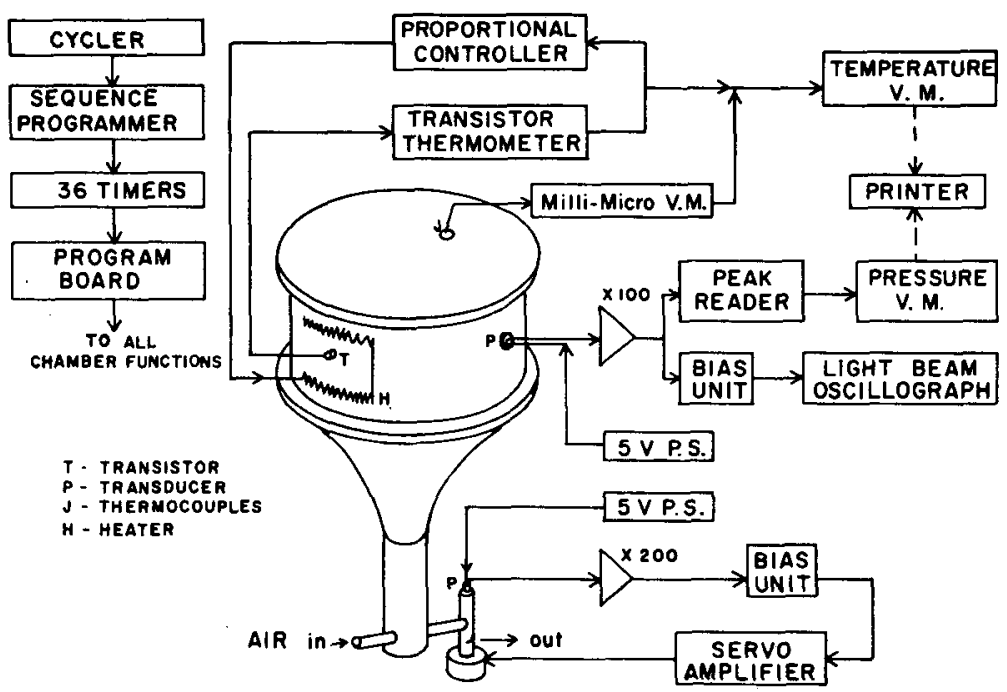

FIG. 2. Diagram of systems which operate the chamber and measure and control the pressure and temperature.

similar to past procedures. The carrier gas is inserted through a valve in the side of the chamber and the chamber is flushed several times with the carrier gas. As will be discussed later the introduction of the carrier gas was found to be the source of a major interfering impurity. Cleaning expansions (first employed by Wilson), similar to the data expansion but to only intermediate supersaturations, are routinely used to remove re-evaporation nuclei (droplets formed in previous expansions which have not fallen into the pool or evaporated) from the chamber by condensing water on them and letting them fall into the liquid pool, a procedure which has been rigorously followed for many years. Generally three or four cleaning expansions are used between data expansions. The underside of the stainless steel piston was milled out leaving radial ribs to provide a lightweight yet stiff piston plate. Its position is controlled by the air pressure in a pneumatic chamber in the lower part of Fig. 1. Its position is never defined by mechanical stops as in a volume ratio chamber. Electro-mechanical brakes are used to dampen piston oscillations where desired. This high performance piston was needed to achieve controlled pulse sensitive times. For normal operation only a short pulse of nucleation on the order of $0.01 \mathrm{~s}$ is allowed, although longer nucleation pulses can be generated with some effort. This short "sensitive time" effectively separates nucleation from droplet growth effects. During the study of anomaly 3 ) the pulse was broadened in discrete steps to investigate the variation of nucleation rate with time which is always definitive proof that a minority heterogeneous nucleant is present. Photography with subsequent drop counting is the primary drop concentration observation method. Closed circuit television, which has less resolution than photography, is used to check in real time that the chamber is working properly without having to enter the environmental enclosure when uncomfortable temperature regimes are being investigated. The cloud chamber plus all the other items shown in Fig. 1 are housed in a temperature-controlled environmental chamber capable of delivering initial temperatures for experiments in the range from 325 to $250 \mathrm{~K}$. Typical expansions have a maximum temperature depression of $30^{\circ} \mathrm{C}$. A typical expansion profile is shown in Fig. 3. Following the photograph of the droplet density during an expansion an overcompression is performed of long enough duration to counter balance the heat flow into the chamber. This procedure minimizes the heat pumping associated with a continuous string of expansions and the thermal anomalies which accompany it.

The nucleation rate $J$ varies rapidly over the nucleation pulse shown in Fig. 3, reaching its maximum when the supersaturation ratio $S$ reaches its maximum. $J$ is obtained in a straightforward manner from the drop count and the time dependent conditions of supersaturation and temperature which produced the drops. Analysis of the short nucleation pulse shows the majority of the nucleation occurs during a time of $\sim 0.01 \mathrm{~s}$ at the peak. The method for calculating $J$ (Schmitt et al., 1981) assumes the classical functional form

$$
J=S^{2} \exp \left[A+B /(\ln S)^{2}\right],
$$

where $A$ and $B$ contain adjustable parameters and depend on the temperature $T$. These parameters are adjusted to obtain the best fit of $\int J(t) d t$ to the measured drop counts. $J$ is a function of time since $S$ and $T$ are time dependent. 


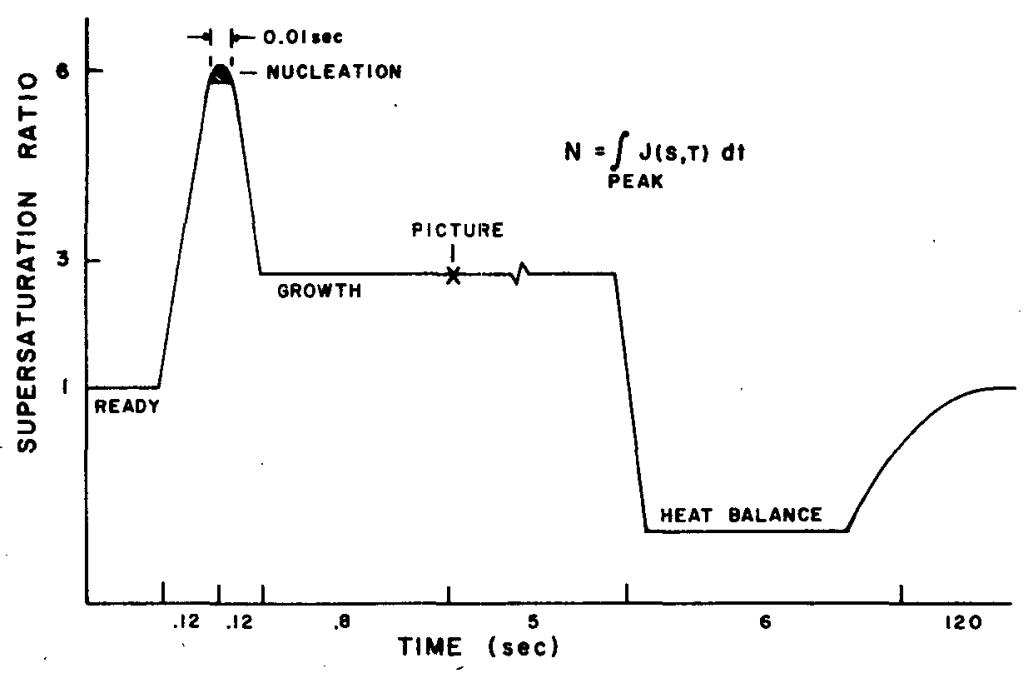

FIG. 3. Typical supersaturation ratio vs time profile.

\section{Experiments}

\section{a. High temperature nucleation exhibiting a "knee"}

The high temperature nucleation experiments of Allen and Kassner (1969) and Biermann (1971) were

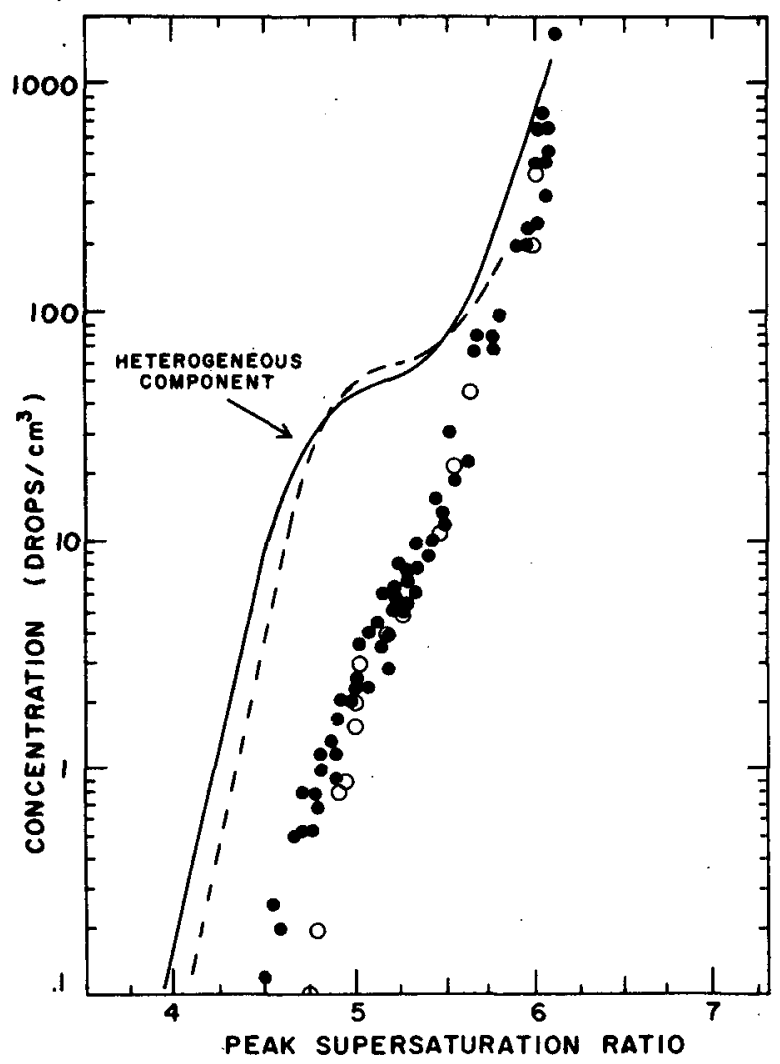

Fig. 4. Comparison of measurements using this facility with previous expansion chamber measurements. Dashed line: Allen and Kassner $(1969), 22.5^{\circ} \mathrm{C}$. Solid line: Biermann $(1971), 25^{\circ} \mathrm{C}$. Closed circle: This work, before high supersaturation cleaning expansions. Open circle: This work, after high supersaturation cleaning expansions. repeated to investigate the anomalous "knee" appearing in their data (see Fig. 4). Classical nucleation theory (Becker-Doring, 1935; Frenkel, 1955) as well as the molecular model for nucleation (Hale and Plummer, 1974) predict a smooth curve, almost a straight line on a semi-log plot of the data as in Fig. 4. This general shape is what is observed in the present data; the conspicuous knee has disappeared. Each point represents the drop concentration and peak supersaturation ratio obtained from one short-pulse expansion. The lines are fits of the data points obtained by Allen and Biermann. While there is excellent agreement between the present and the older data in the upper part of the graph, which represents homogeneous nucleation, the heterogentous component, or knee, in the Allen and Biermann data has now disappeared. The impurity was simply left out in the construction of a new chamber in a different laboratory room. It was likely to have been due to some contaminant found in the confines of a 75-year old laboratory room. To the best of our knowledge similar materials were employed in the ccinstruction of the new chamber. The absence of the heterogeneous component greatly simplifies the analysis of the homogeneous nucleation data.

The data of Allen and Kassner (1969) shows good agreement with earlier cloud chamber data of Volmer and Flood (1934) and that of Powill (1928). Hence the interfering impurity is not something associated only with a modern laboratory, it was also present in some of the very early cloud chlamber experiments.

A slight knee, due to a small number of residual impurities, can still be seen in our data. 'The use of high supersaturation cleaning expansions, to be described in the next section, and the continuous operation of the chamber reduced considerably the already small population of these residual impurities (see Fig. 4).

It should be noted that the observation of the tem- 


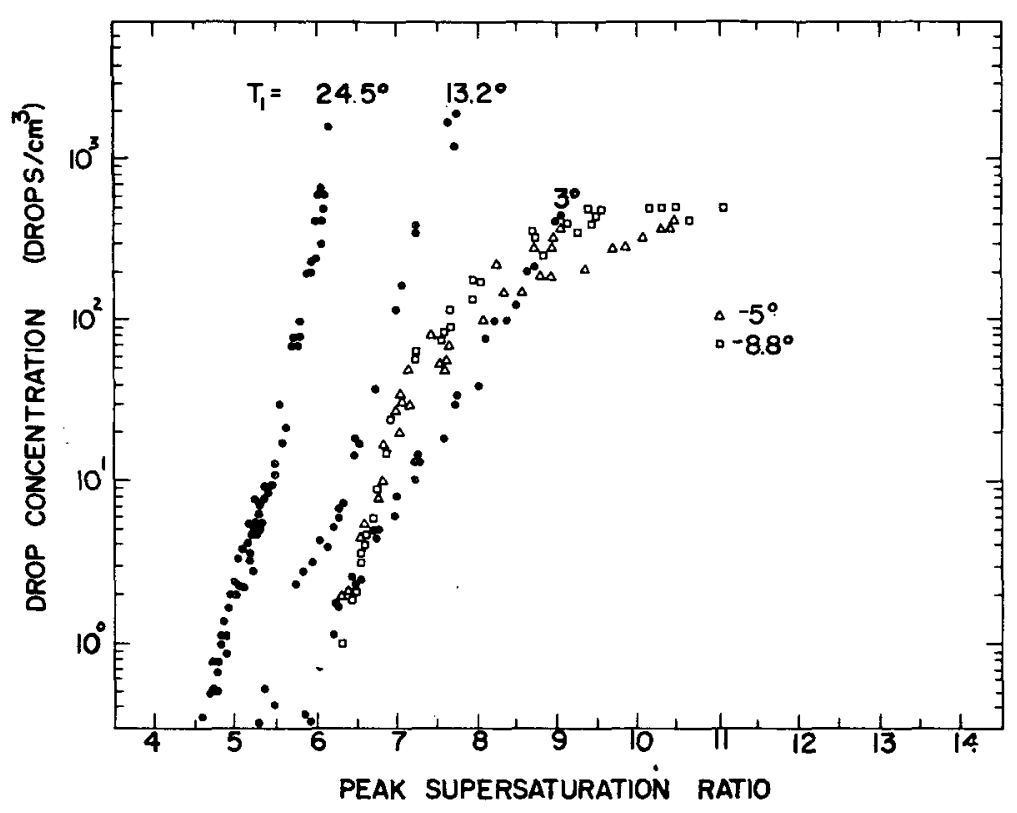

FIG. 5. Preliminary data over a range of pre-expansion temperatures, $T_{1}$.

perature dependence of critical supersaturation does not give any indication of the presence of any anomaly or heterogeneous nucleant. It is not evident until one makes measurements over a considerable range of nucleation rates, and perhaps not then unless the heterogeneous nucleant is present in concentrations of $\sim 1-10^{3} \mathrm{~cm}^{-3}$. Substantially larger concentrations would not yield a knee because the population of heterogeneous centers would not be substantially depleted (see Section 3 which describes the observation of the decay of the nucleation rate with time).

Because certain active impurities are occasionally found in gas-vapor systems in such minute concentrations, is it possible to rain-out these species by simply running the chamber continuously at supersaturations which would be expected to nucleate on the bulk of these impurities. We believe that the large liquid pool at the floor of the chamber is of great value in trapping these impurities and reducing the rate at which they make their way back into the sensitive volume. Although the mechanism of the process is unclear, it is observed that when the chamber is left idle for long periods of time, the sensitive volume does become repopulated with them.

Similar effects have been observed in thermal diffusion cloud chambers (Katz, 1970). The continuous operation of these chambers increases the rate at which impurities that are active at a given level of supersaturation can be rained out. However, these impurities very obviously exhibit a distribution of critical supersaturations and it would appear that the expansion cloud chamber's ability to operate at higher peak supersaturations is an advantage in cleaning out the entire distribution of active impurities. Moreover, the large liquid pool in the base of the chamber should be more effective in retaining the impurity than the small amount of liquid used to wet the plates of thermal diffusion cloud chambers. In all probability the two types of cloud chambers possess nearly equal characteristics in as far as their ability to sweep out impurity constituents is concerned.

\section{b. Temperature-dependence of the critical super- saturation at low temperature}

Madonna et al. (1961) observed an interesting phenomenon. As temperature decreases the critical supersaturation required for the onset of homogeneous nucleation would be expected to increase, on the basis of classical theory. This behavior as the temperature decreased was observed until a temperature near $255 \mathrm{~K}$ and a supersaturation near 5.6 was reached. Then as the temperature was further lowered, the onset of nucleation continued to occur at a supersaturation of 5.6. Madonna suggested that this could be an indication of a heterogeneous component in the nucleation.

The same phenomenon was observed in the early low temperature experiments in our new chamber. Fig. 5 shows the drop concentration vs supersaturation ratio for short-pulse expansions at various initial temperatures, $13.2,3,-5$, and $-8.8^{\circ} \mathrm{C}$. The important observation, that the -5 and $-8.8^{\circ} \mathrm{C}$ data were practically identical to those for $3^{\circ} \mathrm{C}$, was a clear indication that an impurity, similar to that observed by Madonna et al. (1961), had appeared which would serve to mask the observation of homogeneous nucleation at low temperatures. This impurity began to activate at a critical supersaturation ratio around 5.6 at temperatures below $-15^{\circ} \mathrm{C}$ and increased to a maximum concentration of $500 \mathrm{~cm}^{-3}$ 


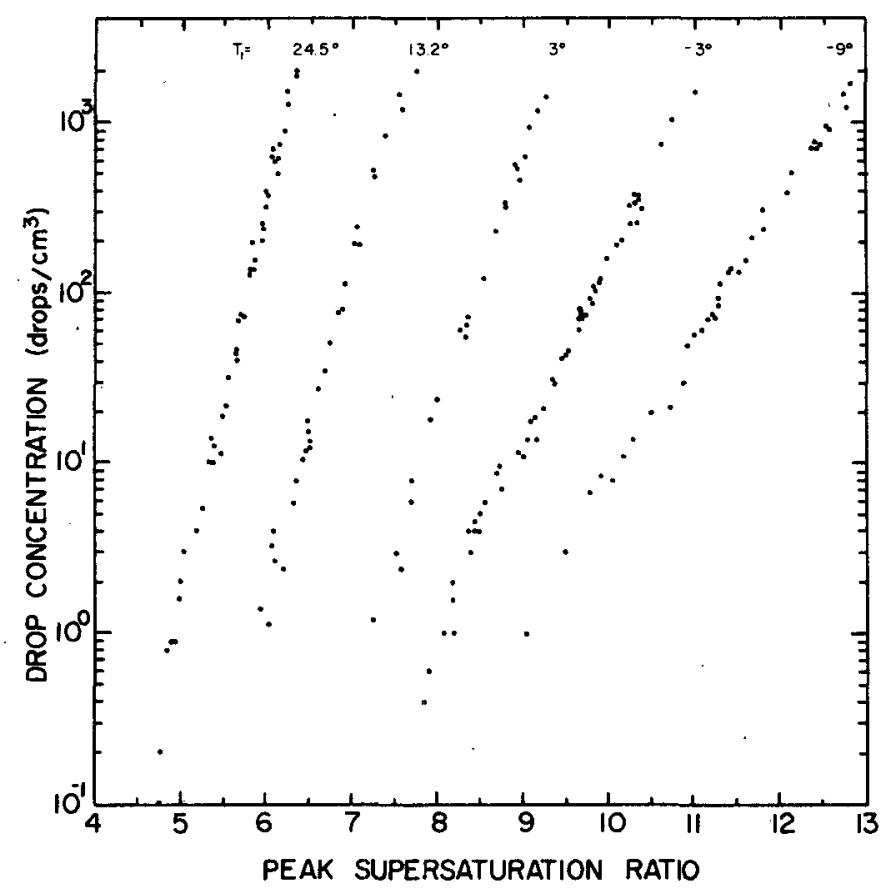

FIG. 6. Drop concentration vs supersaturation after using high supersaturation cleaning expansions.

(see Fig. 5). This behavior seemed to be independent of temperature as the temperature was further lowered.

The source of and a solution to this impurity prob-

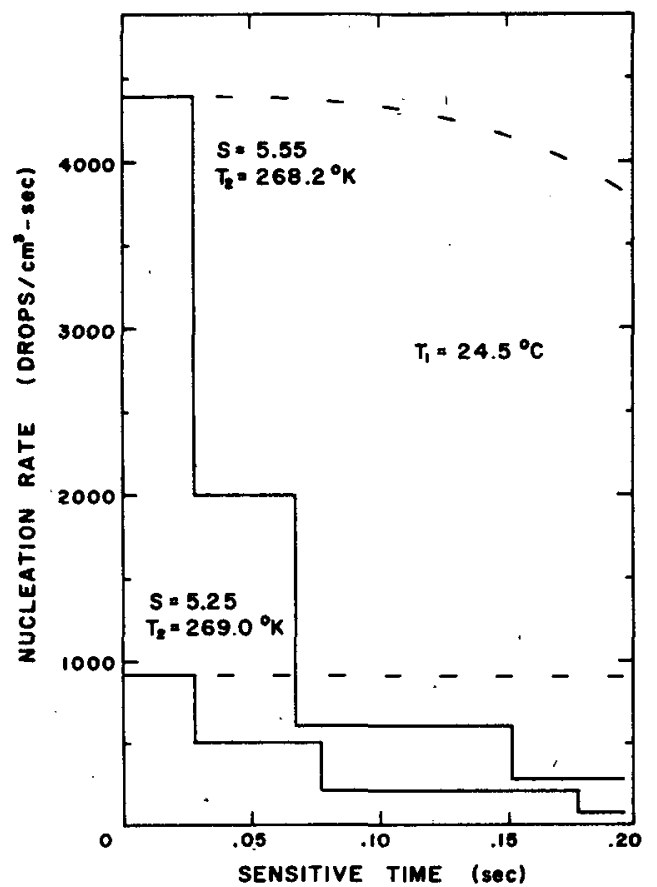

Fig. 7. Preliminary nucleation rate measurements as functions of time for constant supersaturation, $S$, and temperature, $24.5^{\circ} \mathrm{C}$ data set. lem were found. Experiments revealed that numerous cleaning expansions to supersaturations exceeding those reached during subsequent data expansions could deplete the impurity. Normally the routine cleaning expansions are limited to supersaturations less than that used in the data expansion. During the high supersaturation cleaning expansions, droplets formed on the heterogeneous centers which were previously activated only during actual data taking. These droplets with the heterogeneous centers trapped inside fell from the sensitive volume to the liquid pool at the floor of the chamber and were thus removed. Subsequent experiments showed that following such a procedure (numerous high supersaturation cleaning expansions), the heterogeneous component failed to reappear during normal chamber operation unless new argon (the carrier gas) was introduced into the chamber. The source of the impurity was therefore either the argon tank, the regulator, or the PVC plastic tubing used to connect the gas bottle to the chamber. PVC tubing is suspect due to the volatility and high concentration of plasticisers such as dioctyl phthalate used in it, and the argon gas may also contain oil vapors originating in the compressors used to pump the gas into the tank.

Fig. 6 shows the drop concentration vs supersaturation ratio for short pulse expansions at various initial temperatures made after high supersaturation cleaning expansions were used to remove the impurities. Comparison of this data with that shown in Fig. 5 reveals that now the critical supersaturation (for the onset of nucleation) continues to rise as the 
temperature is lowered; it does not reach 5.6 and then stop as in Fig. 5. Small knees can still be seen in some of the data where our techniques for suppressing the heterogeneous component discussed earlier were not completely effective. However, even in the case of these data the number of heterogeneous centers was quite small and did not mask the validity of homogeneous nucleation data being taken.

It is important to note here that we are dealing with two distinct types of heterogeneous nucleation centers. Each has its own distinct characteristics. The first is active at all temperatures and produces only a relatively small and nearly constant decrease in the free energy of formation as compared with that for homogeneous nucleation (see Allen and Kassner, 1969). The second appears to require some low temperature as a threshold and at temperatures below this it becomes increasingly active. Moreover, it is not clear from the data taken whether the supersaturation or the temperature was the primary stimulant. The threshold temperature for the appearance of ice, $-40^{\circ} \mathrm{C}$, was not reached at this time, so the droplets were supercooled water (Anderson et al., 1980). Again, the data seem to indicate that a distribution of activities were present.

\section{c. Decay of the nucleation rate with time}

Classical theory (Becker-Doring, 1935; Frenkel, 1955; Abraham, 1974) predicts that the nucleation rate should be constant if the supersaturation is constant. However, Allard and Kassner (1965), Allen

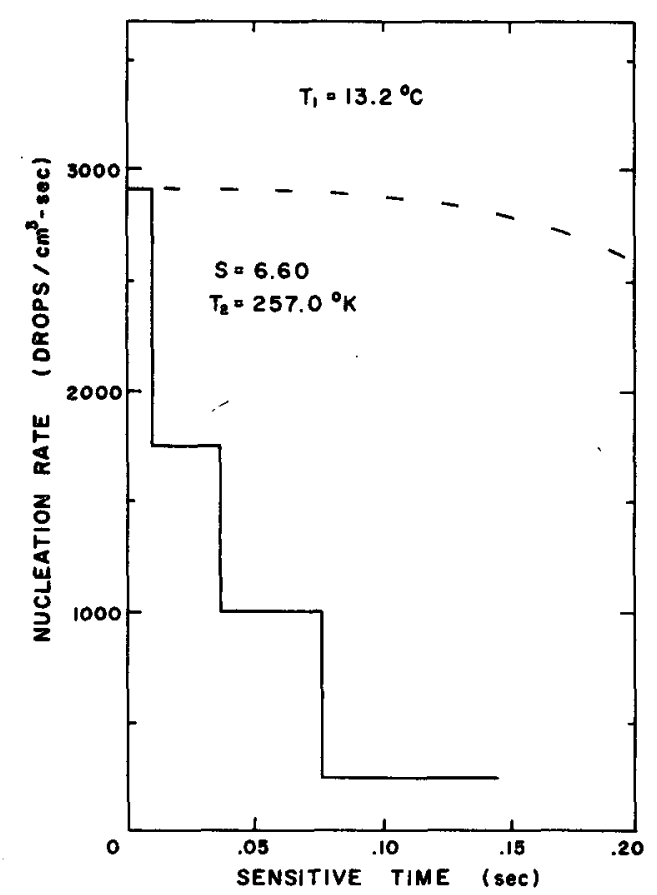

Fig. 8. Preliminary nucleation rate measurements as functions of time for constant supersaturation, $S$, and temperature, $13.2^{\circ} \mathrm{C}$ data set.

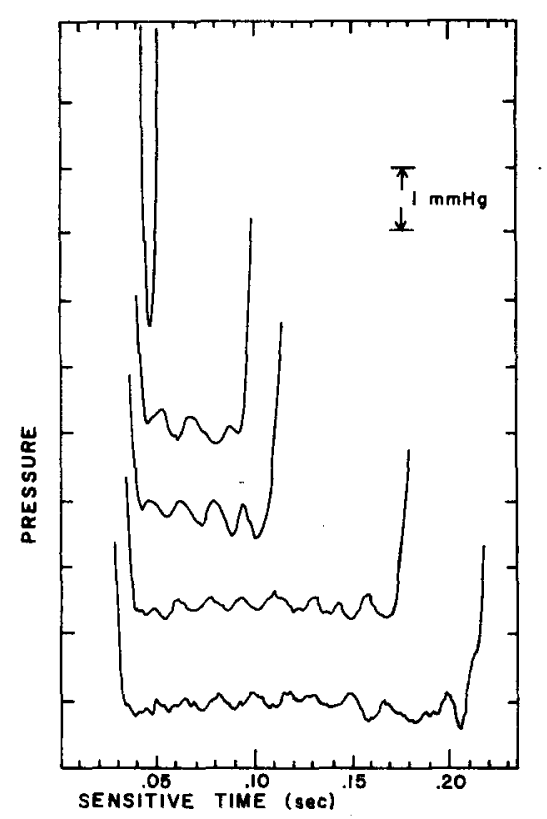

Fig. 9. Pressure at the peak of the expansion for different sensitive times.

and Kassner (1969) and Biermann (1971) all observed a nearly exponential decay of the nucleation rate with time. They attributed the decay to the existence of heterogeneous impurities which were depleted as the nucleation proceeded. Figs. 7 and 8 show nucleation rates measured with the present chamber before the abovementioned high supersaturation cleaning expansions were inaugurated. The various sensitive times (time of constant supersaturation during which nucleation occurs) were achieved by auxiliary small expansion valves after the main expansion valve closes so as to just offset the recompressive effect due to the heat influx from the walls. At the end of the desired sensitive time the normal partial recompression is negotiated in order to terminate the nucleation but still allow for rapid droplet growth. The net effect is to widen the nucleation pulse; the narrow version of which is shown in Fig. 3. The rest of the chamber cycle remains the same. Fig. 9 shows examples of the expansion profiles (pressure vs time in the region near the pressure minimum) used for these measurements. Figs. 7 and 8 exhibit the same exponential decay of the nucleation rate with time that was previously observed (Allard and Kassner, 1965; Allen and Kassner, 1969; Biermann, 1971). The dashed lines indicate the behavior expected of the nucleation when the supersaturation depletion due to drop growth is taken into account. This effect does not account for the observed decay in the nucleation rate. The decay rates are obtained by subtracting the drop count for a longer pulse from that for the next shorter pulse.

Results of nucleation rate measurements as functions of time using the variable wide-pulse technique, 


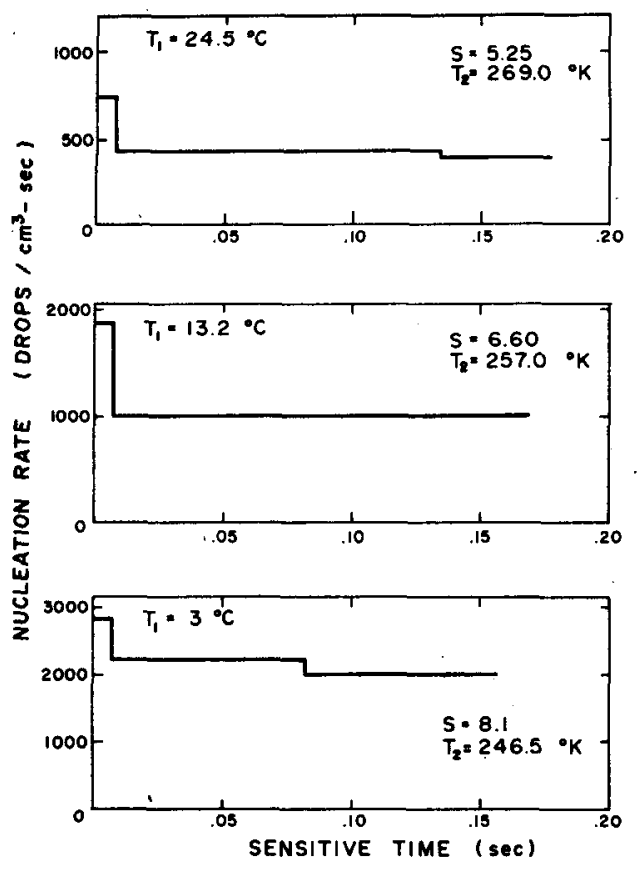

FIG. 10. The nucleation rate measured as a function of time at constant supersaturation, $S$, and temperature, $T_{2}$

after inaugurating the high supersaturation cleaning expansions, are shown in Fig. 10 for initial temperatures of $24.5,13.2$ and $3^{\circ} \mathrm{C}$. These measurements show that after an initially somewhat higher value, attributed to nucleation on a few impurities which can be subtracted out as a background count, the nucleation rate did not show the decay with time that was previously observed for similar temperature and supersaturation conditions. The total heterogeneous nuclei concentration, due to ions, impurities and reevaporation nuclei, is given by the product of the width and height of this initial bump $(\Delta J \cdot \Delta t)$ and amounts to $\sim 5 \mathrm{~cm}^{-3}$. This is the first time that the nucleation rate in an expansion chamber was observed to remain constant in time and is, therefore, the first evidence of this type to support the contention that homogeneous nucleation was indeed being observed in the expansion chamber. Also these results demonstrate the extreme sensitivity of the widepulse technique in detecting small levels of heterogeneous nuclei. We may conclude that the observation of a constant nucleation rate with time is a necessary but not sufficient condition that homogeneous nucleation is being observed, because it is always possible that the concentration of heterogeneous centers is so large that the decay is imperceptible in this type of observation.

A small discrepancy needs to be noted. The nucleation rates measured during the later time periods in the earlier $24.5^{\circ} \mathrm{C}$ data (Fig. 7) for both $S=5.25$ and 5.55 were lower than the homogeneous rate measured in the clean (after the high supersaturation cleaning expansions) chamber (Fig. 10) for the same $25^{\circ} \mathrm{C}$ and $S=5.25$. Obviously the rates in the earlier data should be at least that of homogeneous nucleation. We believe that the vapor depletion due to the growth of the large number of heterogenersusly nucleated drops evident in Fig. 7 was sufficient to raise the temperature and reduce the supersaturation by the small amounts necessary so that homogeneous nucleation was absent during the later time periods of the wide pulse. These effects were pronounced because growth on heterogeneous centers began even before the peak supersaturation of the wide pulse was reached and the centers were already larger than the critical embryo size for homogeneous nucleation. The slight decay observed in the graphs presented in Fig. 10 are consistent with the probable; error of the experiment and the expected vapor depletion by growing droplets.

\section{d. The nature of the impurity}

The anomalous behavior of the nucleation rate observed as a knee by Allard and Kassner (1965), Allen and Kassner (1969), and Biermann (1970) has been the subject of much speculation. Allen and Kassner (1969) suggested that it might possibly be due to the incorporation of one molecule of $\mathrm{H}_{2} \mathrm{O}_{2}$ into the cluster structure of the prenucleation embryo because the impurity seemed to displace the nucleation rate curve (see Fig. 4) by a nearly constant amount to the left. They surmized that the impurity was most likely due to some naturally occurring mechanism which might involve cosmic rays and natural radioactivity as the instigating fac:or. $\mathrm{H}_{2} \mathrm{O}_{2}$ could be one possible compound formed by energetic ionizing radiation. In order to check the validity of the $\mathrm{H}_{2} \mathrm{O}_{2}$ hypothesis, Biermann added a quantity of $\mathrm{H}_{2} \mathrm{O}_{2}$ to the water in the base of the cloud chamber. The nucleation rate was observed to increase to an indeterminable high value which subsequently diminished. The chamber eventually becami cleaner than before the introduction of the $\mathrm{H}_{2} \mathrm{O}_{2}, \mathrm{H}_{2} \mathrm{O}_{2}$ is an effective oxidant which could have easily reacted with trace organics to produce the observed effect. Stoddard (1977) used the new cloud chamber facility described in this paper to study the mobility of ion clusters at $100 \%$ relative humidity. He uised $\mathbf{P o}^{210}$, an alpha particle emitter, as a source of ionizing radiation. The alpha particles passed through a thin mica window and into the ion source region of the chamber. It was found that neutral background built up in the chamber to unacceptable levels when the alpha particles bombarded the chamber continuously. Ion-molecule reactions no doubt account for the observed heterogeneous nuclei. Stodiard also found that if he passed the argon gas, used in the chamber sensitive volume, through an activated charcoal filter maintained somewhat above liquid nitrogen temperature, the impurity which resulted in the unwanted species was virtually eliminated. 
Actually the argon was condensed into the charcoal and the charcoal trap subsequently warmed up enough to evaporate the argon into the chamber. In this way intimate contact between the argon and the activated charcoal could be insured. The charcoal was activated in an oven under vacuum prior to use. This procedure indicated that the impurity most likely accompanied the argon and could be oil vapors originating from compressors used to pump the argon into the tanks by the supplier. The current evidence favors ion-molecule reactions as the agent responsible for at least one of the heterogeneous constituents described in this paper.

\section{Conclusions}

The new and improved expansion cloud chamber, which is relatively free of impurities capable of serving as heterogeneous nuclei, has been used to resolve three nucleation anomalies: the knee in the drop concentration vs supersaturation data of Allen and Kassner (1969) and Biermann (1971); the temperature dependence of the critical supersaturation data observed by Madonna et al. (1961); and the exponential decay of the nucleation rate with time observed by Allard and Kassner (1965), Allen and Kassner (1969), and Biermann (1971).

In all cases the anomalies were associated with heterogeneous nucleation on impurities interfering with the desired homogeneous nucleation. Removal of the impurities was accomplished by judicious selection of materials for chamber construction and by the use of routine low supersaturation cleaning expansions between data expansions. The employment of periodic high supersaturation cleaning expansions successfully dealt with an impurity particularly active at low temperatures.

The use of wide expansion pulses in which the length of time during which nucleation occurred was controlled and varied was found to be an extremely sensitive method for determining the impurity levels in the cloud chamber.

Acknowledgments. This material is based on work supported by the Division of Atmospheric Sciences, National Science Foundation under Grant ATM7919480.

\section{REFERENCES}

Abraham, F. F., 1974: Homogeneous Nucleation Theory. Academic Press, 263 pp.

Allard, E. F., and J. L. Kassner, Jr., 1965: New cloud chamber method for the determination of homogeneous nucleation rates. J. Chem. Phys., 42, 1401-1405.

Allen, L. B., and J. L. Kassner, Jr., 1969: The nucleation of water vapor in the absence of particulate matter and ions. J. Colloid Interface Sci., 30,81-93.

Anderson, R., R. Miller, J. L. Kassner, Jr. and D. Hagen, 1980: A study of homogeneous condensation-freezing nucleation of small water droplets in an expansion cloud chamber. J. Atmos. Sci., 37, 2508-2520.

Becker, R., and W. Doring, 1935: Kinetic theory of nucleation in a supersaturated vapor. Ann. Phys., 24, 719-729.

Biermann, A. H., 1971: Homogeneous nucleation of water vapor in inert gas atmospheres. Ph.D. dissertation, University of Missouri-Rolla, 59 pp.

Das Gupta, N. N., and S. K. Gnosh, 1946: A report on the Wilson cloud chamber and its application in physics. Rev. Mod. Phys., 18, 225-290.

Frenkel, J., 1955: Kinetic Theory of Liquids. Dover, 488 pp.

Hale, B. N., and P. L. M. Plummer, 1974: On nucleation phenomena I: A molecular model. J. Atmos. Sci., 31, 1615-1621.

Hirth, J. P., and G. M. Pound, 1963: Condensation and Evaporation. Macmillan, $191 \mathrm{pp}$.

Kassner, J. L., Jr. and R. L. Schmitt, 1966: Homogeneous nucleation measurements of water vapor in helium. J. Chem. Phys., 44, 4166-4170.

- J. C. Carstens and L. B. Allen, 1968: Analysis of the heat and vapor propagation from the walls of the Nolan, Pollak, and Gardner type condensation nucleus counter. J. Atmos. Sci., 25, 919-926.

Katz, J. L., 1970: Condensation of a supersaturated vapor. I. The homogeneous nucleation of the $n$-alkanes. J. Chem. Phys., 52, 4733-4748.

Madonna, L., C. Sciulli, L. Canjar and G. M. Pound, 1961: Low temperature cloud chamber studies on water vapor. Proc. Phys. Soc., 78, 1218-1222.

Powell, C. F., 1928: Condensation phenomena at different temperatures. Proc. Roy. Soc. London, A119, 553-577.

Schmitt, J. L., 1981: A precision expansion cloud chamber for homogeneous nucleation studies. Rev. Sci. Instrum., 52, 10914.

- G. W. Adams and R. A. Zalabsky, 1981: Homogeneous nucleation of ethanol. J. Chem. Phys. (in press).

Stoddard, L. E., 1977: Measurements of the mobilities of small aqueous ions in argon saturated with water vapor using an expansion cloud chamber. Ph.D. dissertation, University of Missouri-Rolla, $103 \mathrm{pp}$.

Volmer, M., and H. Flood, 1934: Tropfchenbildung in dampfen. Z. Phys. Chem. (Leipzig), A170, 273-285.

Wilson, C. T. R., 1897: Condensation of water vapor in the presence of dust-free air and other gases. Phil. Trans. Roy. Soc. London, 189, 265-307. 